\section{FRI0165 PATIENT- AND PHYSICIAN-REPORTED OUTCOMES FROM RAJ4: A RANDOMISED, DOUBLE-BLIND, PLACEBO-CONTROLLED STUDY OF THE EFFICACY AND SAFETY OF PEFICITINIB (ASP015K) IN PATIENTS WITH RHEUMATOID ARTHRITIS (RA) WHO HAD AN INADEQUATE RESPONSE TO METHOTREXATE (MTX)}

Yoshiya Tanaka ${ }^{1}$, Tsutomu Takeuchi ${ }^{2}$, Mitsuhiro Rokuda ${ }^{3}$, Hiroyuki Izutsu ${ }^{3}$, Yuichiro Kaneko ${ }^{3}$, Teruaki Shiomi ${ }^{3}$, Emi Yamada ${ }^{3} .{ }^{1}$ University of Occupational and Environmental Health, Kitakyushu, Japan; ${ }^{2}$ Keio University School of Medicine, Tokyo, Japan; ${ }^{3}$ Astellas Pharma, Inc., Tokyo, Japan

Background: The novel oral Janus kinase inhibitor, peficitinib, demonstrated efficacy and tolerability as once-daily combination therapy with MTX for moderate-to-severe RA in the RAJ4 phase 3 study (NCT02305849). ${ }^{1}$ For the first time, we focussed on patient and physician outcome measures in this Japanese study.

Objectives: To assess health-related quality of life (HRQoL) of Japanese patients with RA who had an inadequate response to MTX.

Methods: This multicentre, randomised, double-blind, parallel-group, placebo-controlled, phase 3 study recruited patients in Japan with active RA and inadequate response to MTX. ${ }^{1}$ Patients were randomised 1:1:1 to 52week placebo+MTX (PBO), peficitinib $100 \mathrm{mg}$ QD+MTX (PEFI $100 \mathrm{mg}$ ) or peficitinib $150 \mathrm{mg}$ QD+MTX (PEFI $150 \mathrm{mg}$ ). The PBO group was switched to PEFI $100 \mathrm{mg}$ or $150 \mathrm{mg}$ at Week 12 (for non-responders) or Week 28 (for remaining patients). Patient- and physician-reported outcome assessments included: Physician's Global Assessment of disease activity (PGA); Subject's Global Assessment of disease activity (SGA); and Subject's Global Assessment of pain (SGAP) by visual analogue scale. Physical function was evaluated using Health Assessment Questionnaire-Disability Index (HAQ-DI). All assessments were conducted at each visit from baseline to the end of study.

Results: 519 patients were treated: PBO ( $n=170)$, PEFI $100 \mathrm{mg}(n=175)$ and PEFI $150 \mathrm{mg}(n=174)$. One PEFI $100 \mathrm{mg}$ patient was excluded from the analysis due to protocol deviation. At Weeks 12/early termination (ET) and 28/ET, both PEFI groups were associated with significant improvements in PGA, SGA, SGAP and HAQ-DI (Table 1). These outcomes were maintained or improved at end of treatment (EOT; Week 52/ ET), when mean change from baseline for PGA, SGA, SGAP and HAQ$\mathrm{DI}$ in the PEFI $100 \mathrm{mg}$ and PEFI $150 \mathrm{mg}$ groups were: -38.41, -43.33; $29.34,-34.05 ;-28.94,-32.68$; and $-0.36,-0.51$, respectively. Significantly greater proportions of patients achieved functional remission and a minimum clinically important $(\mathrm{MCl})$ reduction in HAQ-DI score $(\leq 0.5$ and reduction of $>0.22$, respectively) at Weeks $12 / E T$ and $28 / E T$ in both PEFI groups than in the PBO group $(p<0.001$ vs PBO for both PEFI doses; Table 1). In the PEFI $100 \mathrm{mg}$ and PEFI $150 \mathrm{mg}$ groups at EOT, functional remission occurred in 105/172 (61.0\%) and 106/171 (62.0\%) patients and $\mathrm{MCl}$ reduction in HAQ-DI score occurred in 99/172 (57.6\%) and 120/171 (70.2\%) patients, respectively.

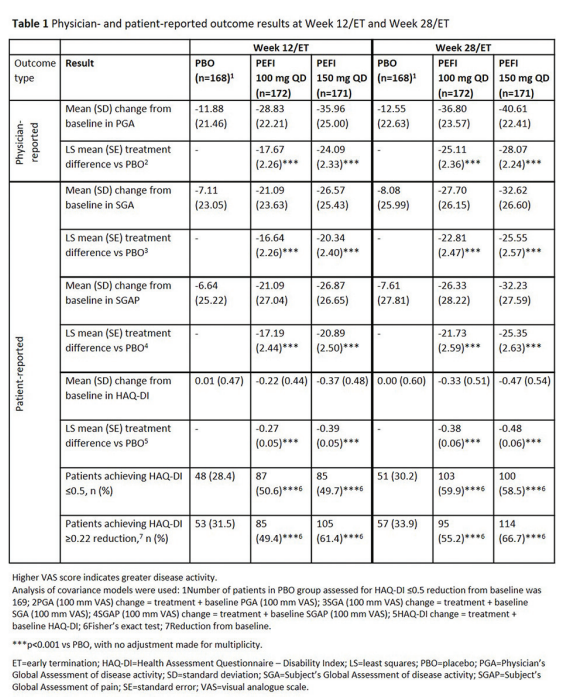

Conclusion: Measures of PGA, SGA, SGAP and HAQ-DI in patients with RA who had an inadequate response to MTX demonstrated improved HRQoL with peficitinib; PEFI $150 \mathrm{mg}$ QD showed similar or numerically greater changes in HRQoL than PEFI $100 \mathrm{mg}$ QD. Improvements were maintained or improved to the EOT and demonstrated a significant benefit for patients with RA in Japan.

\section{REFERENCE:}

[1] Takeuchi T, et al. Arthritis Rheumatol 2018; 70 (Suppl. 10): Abstract 888.

Acknowledgement: This study was sponsored by Astellas Pharma, Inc. Medical writing support was provided by Rhian Harper Owen of Cello Health MedErgy and funded by Astellas Pharma, Inc.

Disclosure of Interests: Yoshiya Tanaka Grant/research support from: Abbvie, Astellas, Bristol-Myers Squibb, Chugai, Daiichi-Sankyo, Eisai, Mitsubishi-Tanabe, MSD, Ono, Taisho-Toyama, Takeda, Speakers bureau: Abbvie, Asahi-kasei, Astellas, Bristol-Myers Squibb, Chugai, Daiichi-Sankyo, Eli Lilly, Eisai, Glaxo-Smithkline, Janssen, Mitsubishi-Tanabe, Novartis, Pfizer Japan Inc, Sanofi, Takeda, UCB, YL Biologics, Tsutomu Takeuchi Grant/research support from: Astellas Pharma Inc, Chugai Pharmaceutical Co, Ltd., Daiichi Sankyo Co., Ltd., Takeda Pharmaceutical Co., Ltd., AbbVie GK, Asahikasei Pharma Corp., Mitsubishi Tanabe Pharma Co., Pfizer Japan Inc., Eisai Co., Ltd., AYUMI Pharmaceutical Corporation, Nipponkayaku Co. Ltd., Novartis Pharma K.K., Grant/research support from: AbbVie, Asahi Kasei, Astellas, AstraZeneca, AYUMI, BristolMyers Squibb, Chugai, Daiichi Sankyo, Eisai, Eli Lilly Japan, Janssen, Mitsubishi Tanabe, Nippon Kayaku, Novartis, Pfizer Japan Inc, Taiho, Taisho Toyama, Takeda, Teijin, Grant/research support from: Astellas Pharma Inc., Bristol Myers Squibb, Chugai Pharmaceutical Co., Ltd., Mitsubishi Tanabe Pharma Co., Pfizer Japan Inc., Santen Pharmaceutical Co., Ltd., Takeda Pharmaceutical Co., Ltd., Teijin Pharma Ltd., AbbVie GK, Asahi Kasei Pharma Corp., Taisho Toyama Pharmaceutical Co., Ltd. SymBio Pharmaceuticals Ltd., Janssen Pharmaceutical K.K., Celltrion Inc., Nipponkayaku Co. Ltd., and UCB Japan, Consultant for: Astra Zeneca K. K., Eli Lilly Japan K.K., Novartis Pharma K.K., Mitsubishi Tanabe Pharma Co., Abbivie GK, Nipponkayaku Co.Ltd, Janssen Pharmaceutical K.K., Astellas Pharma Inc., Taiho Pharmaceutical Co. Ltd., Chugai Pharmaceutical Co. Ltd., Taisho Toyama Pharmaceutical Co. Ltd., GlaxoSmithKline K. K., UCB Japan Co. Ltd., Consultant for: AbbVie, Asahi Kasei, Astellas, AstraZeneca, AYUMI, Bristol-Myers Squibb, Chugai, Daiichi Sankyo, Eisai, Eli Lilly Japan, Janssen, Mitsubishi Tanabe, Nippon Kayaku, Novartis, Pfizer Japan Inc, Taiho, Taisho Toyama, Takeda, Teijin, Consultant for: Astra Zeneca K.K., Eli Lilly Japan K.K., Novartis Pharma K.K., Mitsubishi Tanabe Pharma Co., Asahi Kasei Medical K.K., AbbVie GK, Daiichi Sankyo Co., Ltd., Bristol Myers Squibb, and Nipponkayaku Co. Ltd., Speakers bureau: Astellas Pharma Inc., Bristol Myers Squibb, Chugai Pharmaceutical Co., Ltd., Mitsubishi Tanabe Pharma Co., Pfizer Japan Inc., Santen Pharmaceutical Co., Ltd., Takeda Pharmaceutical Co., Ltd., Teijin Pharma Ltd., AbbVie GK, Asahi Kasei Pharma Corp., Taisho Toyama Pharmaceutical Co., Ltd., SymBio Pharmaceuticals Ltd., Janssen Pharmaceutical K.K., Celltrion Inc., Nipponkayaku Co. Ltd., and UCB Japan, Speakers bureau: AbbVie, Asahi Kasei, Astellas, AstraZeneca, AYUMI, Bristol-Myers Squibb, Chugai, Daiichi Sankyo, Eisai, Eli Lilly Japan, Janssen, Mitsubishi Tanabe, Nippon Kayaku, Novartis, Pfizer Japan Inc, Taiho, Taisho Toyama, Takeda, Teijin, Speakers bureau: AbbVie GK., Bristol-Myers K.K., Chugai Pharmaceutical Co. Ltd., Mitsubishi Tanabe Pharma Co., Pfizer Japan Inc., Astellas Pharma Inc, Diaichi Sankyo Co. Ltd., Eisai Co. Ltd., Sanofi K.K., Teijin Pharma Ltd., Takeda Pharmaceutical Co. Ltd., Novartis Pharma K.K., Mitsuhiro Rokuda Employee of: Astellas Pharma, Inc., Hiroyuki Izutsu Employee of: Astellas Pharma, Inc., Yuichiro Kaneko Employee of: Astellas Pharma, Inc., Teruaki Shiomi Employee of: Astellas Pharma, Inc., Emi Yamada Employee of: Astellas Pharma, Inc.

DOI: 10.1136/annrheumdis-2019-eular.2629

\section{FRI0166 RADIOSYNOVIOTHESIS WITH YTTRIUM90: A CANADIAN EXPERIENCE}

Nadil Zeiadin ${ }^{1}$, Eric Turcotte ${ }^{2}$, Emmanouil Rampakakis ${ }^{3}$, Helena Senta ${ }^{2}$, John Sampalis ${ }^{3}$, Carter Thorne ${ }^{1} .{ }^{1}$ Southlake Regional Health Centre, Newmarket, Canada; ${ }^{2}$ University of Sherbrooke, Rheumatology, Sherbrooke, Canada; ${ }^{3} \mathrm{JSS}$ Research, Montreal, Canada

Background: Radiosynovectomy (RSO) has been used since 1952 for treatment of refractory synovitis. ${ }^{90}$ Yttrium in a colloidal form has been approved in several countries as a RSP radiopharmaceutical agent for intra-articular treatment of synovial hypertrophy. In comparison to surgical synovectomy, RSO produces equivalent results, costs less, allows the patient to remain ambulatory and can be repeated. However, there are currently no RSO radiopharmaceuticals approved in Canada.

Objectives: 1. Establish safety of Yttrium-90 citrate synovectomy in a Canadian cohort

2. Determine the therapeutic value of Yttrium-90 citrate in patients with refractory synovitis in a Canadian cohort. 
Methods: Adult $(\geq 18 y 0)$ patients with symptomatic, refractory inflammatory mono- or oligoarthritis were included for RSO with Yttrium-90 citrate as part of a phase-III, prospective, open-label non-controlled trial. All patients were required to have failed 6-months of medical therapy and 2 intraarticular injections and have minimal evidence of cartilage or bone destruction. Only large and medium-sized joints were included (i.e. knees, ankles, wrists and elbows). The dose of Yttrium was adjusted based on the size of joint. Follow-up evaluations were done at 3, 6 and 12 months after RSO. Safety was assessed by patient and clinician reported adverse events. Clinical response was measured by improvement in joint tenderness, effusion and range of motion.

Results: A total of 74 patients and 83 joints ( $88 \%$ knees) were treated with Ytrium-90 citrate. The underlying diagnosis included $25.7 \%$ RA, 34\% SpA, $11 \%$ JIA, and $30.3 \%$ other inflammatory arthritis. Complications included 3 post-RSO flares, 1 septic joint and 2 injection site skin infections. Joint tenderness was reported in $93.8 \%$ of joints at baseline, compared to $50.0 \%$ at $3 \mathrm{mo}(\mathrm{p}<0.001), 55.6 \%$ at $6 \mathrm{mo}(\mathrm{p}<0.001)$ and $40.4 \%$ at $12 \mathrm{mo}(\mathrm{p}<0.001)$. Joint effusion was present in $95.1 \%$ of joints at baseline, $44.3 \%$ at $3 \mathrm{mo}(\mathrm{p}<0.001), 51.4 \%$ at $6 \mathrm{mo} \quad(\mathrm{p}<0.001)$ and $47.4 \%$ at $12 \mathrm{mo}(\mathrm{p}<0.001) .73 .9 \%$ of joints had improvement in range of motion at $3 \mathrm{mo}, 55.9 \%$ at $6 \mathrm{mo}$ and $60.7 \%$ at $12 \mathrm{mo}$.

Conclusion: These results confirm the clinical efficacy and safety of Yttrium90-citrate RSO for refractory synovitis with a sustained clinical benefit at 12 months. This is the first such study in a Canadian cohort. RSO with Ytrium is a safe alternative to surgical synovectomy in refractory cases.

Disclosure of Interests: Nadil Zeiadin: None declared, Eric Turcotte: None declared, Emmanouil Rampakakis : None declared, Helena Senta: None declared, John Sampalis Employee of: JSS Medical Research, Carter Thorne Grant/research support from: Investigator-initiated studies: Amgen, Pfizer. RCTs: Abbvie, Celgene, CaREBiodam, Novartis, Pfizer, Consultant for: Advisory board: Abbvie, Amgen, Celgene, Lilly, Medexus/Medac, Merck, Novartis, Pfizer, Sanofi. Consultant: Abbvie, Centocor, Janssen, Lilly, Medexus/Medac, Pfizer, Speakers bureau: Medexus/Medac

DOI: 10.1136/annrheumdis-2019-eular.431

\section{FRI0167 DOES BARICITINIB HAVE A SPECIFIC EFFECT PATIENT'S REPORTED OUTCOME? COMPARISON OF EFFECTS ON PAIN AND PATIENT GLOBAL ASSESSMENT WITH TNF INHIBITORS}

Eiji Torikai ${ }^{1}$, Daisuke Suzuki ${ }^{2} .{ }^{1} /$ wata city hospital, Iwata, Japan; ${ }^{2}$ Hamamatsu university school of medicine, Hamamatsu, Japan

Background: Baricitinib (bari) is approved for treating moderate-severe rheumatoid arthritis (RA) in many countries, including Japan. Bari is an oral Janus kinase (JAK1)/JAK2 selective inhibitor and has shown good efficacy in RA and an adequate response to conventional synthetic DMARDs in some clinical trials. Pain and patient global assessment (PGA) are common symptoms for patients with RA. Some trials suggest that bari may have a supplementary benefit on patients' reported outcomes (PRO). Our goal was to determine the impact of each treatment on pain and $P G A$.

Objectives: We evaluated and compared the impact of bari and TNF inhibitors (TNFi) on pain and PGA and correlation with PRO and clinical assessments for Japanese RA patients who have an inadequate response to csDMARDs in real world multicenter clinical data.

Methods: We included 60 Japanese RA patients with an inadequate response to csDMARDs. We classified patients into two groups, one treated with bari (B group; 24 females and 8 males) and the other with TNFi (TNFi group; 22 females and 6 males). Patients were scheduled to receive TNFi (GLM $50 \mathrm{mg} / \mathrm{month}$ or ETN $50 \mathrm{mg} /$ week) in TNFi group or bari 4 or $2 \mathrm{mg} /$ day in $\mathrm{B}$ group as a monotherapy or in combination with csDMARDs. We evaluated swollen joint counts on 28 joints (SJC), tender joint counts on 28 joints (TJC), visual analog scale of pain (VAS pain) $(0-100 \mathrm{~mm})$, PGA $(0-100 \mathrm{~mm})$, and Clinical Disease Activity Index (CDAI) at baseline, 4 weeks, and 12 weeks. First, we compared the changes in SJC, TJC, CDAI, VAS pain, and PGA at 4 and 12 weeks after treatments between the two groups. Second, we evaluated the correlation between PROs (VAS pain or PGA) and variations in clinical evaluation items (SJC, TJC, and CDAI).

Results: There were no significant differences in background at baseline between the two groups (Table 1). Baseline SJC, TJC, and CDAI scores; VAS pain; and PGA were $6.67 \pm 3.81,7.93 \pm 3.69,26.35 \pm 8.77,61.2$ \pm 21.8 , and $62.2 \pm 18.4$ in the bari group, respectively, and $6.13 \pm$ $3.21,7.27 \pm 3.56,24.65 \pm 9.72,63.9 \pm 22.7$, and $64.2 \pm 18.4$ in the TNFi group, respectively. SLC, TJC, CDAI scores, VAS pain, and PGA in both groups were significantly improved 1 month after treatment. This tendency continued for 3 months after treatment. There were no significant differences in the improvement rate of SJC, TJC, CDAI scores, VAS pain, and PGA between the two groups at each follow-up time point after treatment. VAS pain scores were significantly correlated with SJC and CDAl scores in both groups (B group; $r=0.651, p<0.01$ and TNFi group; $r=0.688, p<0.01)$. PGA scores were significantly correlated with SJC and CDAl scores in both groups (B group; $r=0.657, p$ $<0.01$ and TNFi group; $r=0.613, p<0.01$ ).

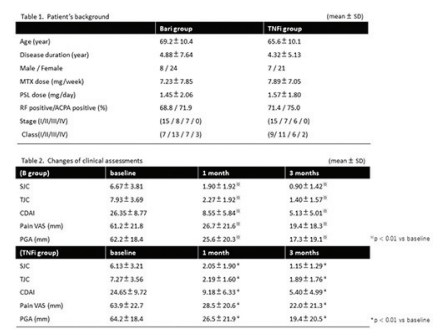

Conclusion: Our results indicate that the effect of bari on pain and PGA is correlated with the improvement rate in disease activity. Bari treatment is not significantly different than TNFi, at least during the short term, in real world clinical data.

\section{REFERENCE:}

[1] Perricone C, et al. Rheumatology (Oxford) 2012;51:866-73

Disclosure of Interests: None declared

DOI: 10.1136/annrheumdis-2019-eular.4515

\section{FRI0168 POSITIVE EFFECTS OF AN ANTI-INFLAMMATORY DIET IN RHEUMATOID ARTHRITIS}

Anna Vadell, Erik Hulander, Linnea Barebring, Inger Gjertsson, Helen Lindqvist, Anna Winkvist. Institute of Medicine, Gothenburg, Sweden

Background: Many patients with rheumatoid arthritis (RA) experience symptom relief of certain foods and earlier research has shown positive effects of food and food components on clinical outcomes of RA. Food components may potentiate each other in their effects on RA, but even so, studies combining foods and food components with probable effects on RA are scarce. Furthermore, comorbidities are common in RA and the risk of cardiovascular disease is elevated in this group. Assumed mechanisms include classical risk factors such as systemic inflammation, dyslipidemia as well as an unfavorable body composition.

Objectives: We investigated if a diet combining foods with anti-inflammatory effects, i.e. an anti-inflammatory portfolio diet, could reduce RA disease activity, markers of inflammation, body composition and blood lipid levels compared to a control diet.

Methods: In this controlled cross-over trial, 50 patients with RA were randomized to start with either intervention or control diet for ten weeks followed by a wash-out period of four months. The intervention diet was an anti-inflammatory portfolio diet containing foods rich in omega-3 fatty acids, fibers and probiotics. The control diet was a typical Swedish diet. Food equivalent to $50 \%$ of daily energy needs was delivered home weekly. For the remaining intake, participants were instructed to consume similar foods as the study diet regimen. Primary outcome measure was change in DAS28, using ESR. Secondary outcomes included changes in blood lipid levels, ESR and CRP as well as body composition.

Results: No significant difference in DAS28 between the groups was seen using Mixed Models analyze $(p=0.116)$. However, DAS28 was significantly lower after the intervention diet compared to after control diet $(p=0.04)$ and a significant reduction over time was seen in the intervention group $(p=0.012)$ using Wilcoxon signed rank test. HDL-cholesterol was increased and TG were lowered after intervention compared to control, analyzed using Mixed Models ( $p=0.046$ and $p=0.006$ respectively). In addition, there was a non-significant trend towards lowered LDL-cholesterol $(p=0.077)$. Comparing after intervention to after control, there were non-significant lower weight, BMI and fat mass after the intervention $(p=0.077, p=0.075$ and $p=0.058$ respectively). None of the other outcomes changed significantly between groups. 